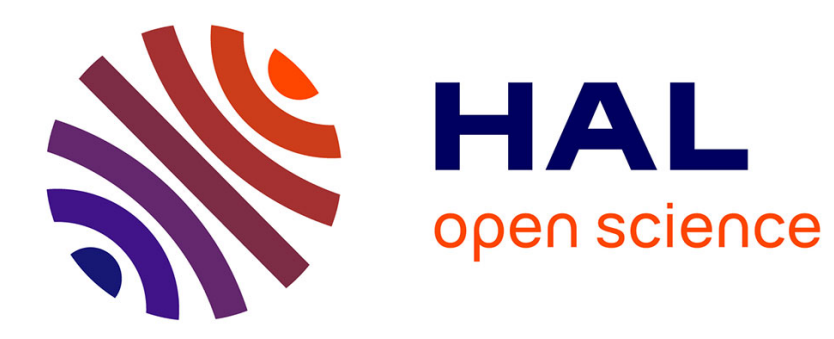

\title{
Strategic manipulability of self-selective social choice rules
}

Mostapha Diss

\section{To cite this version:}

| Mostapha Diss. Strategic manipulability of self-selective social choice rules. 2013. halshs-00785366

\section{HAL Id: halshs-00785366 \\ https://shs.hal.science/halshs-00785366}

Preprint submitted on 6 Feb 2013

HAL is a multi-disciplinary open access archive for the deposit and dissemination of scientific research documents, whether they are published or not. The documents may come from teaching and research institutions in France or abroad, or from public or private research centers.
L'archive ouverte pluridisciplinaire HAL, est destinée au dépôt et à la diffusion de documents scientifiques de niveau recherche, publiés ou non, émanant des établissements d'enseignement et de recherche français ou étrangers, des laboratoires publics ou privés. 
Strategic manipulability of self-selective social choice rules

Mostapha Diss

February 2013 


\section{GATE Groupe d'Analyse et de Théorie Économique Lyon-St Étienne}

93, chemin des Mouilles 69130 Ecully - France

Tel. +33(0)4 72866060

Fax $+33(0) 472866090$

6, rue Basse des Rives 42023 Saint-Etienne cedex 02 - France

Tel. +33 (0)4 77421960

Fax. +33 (0)4 77421950

Messagerie électronique / Email : gate@gate.cnrs.fr

Téléchargement / Download : http://www.gate.cnrs.fr - Publications / Working Papers 


\title{
STRATEGIC MANIPULABILITY OF SELF-SELECTIVE SOCIAL CHOICE RULES
}

\author{
Mostapha Diss* \\ This version, December 2012
}

\begin{abstract}
We provide exact relations giving the probability of individual and coalitional manipulation of three specific social choice functions (Borda rule, Copeland rule, Plurality rule) in three-alternative elections when the notion of self-selectivity is imposed. The results suggest that the Borda rule is more vulnerable to coalitional manipulation than the Copeland rule and the Plurality rule. However, Plurality rule seems to be more vulnerable to individual manipulability when the number of voters is greater than a certain threshold value. In addition, the probability of individual and coalitional manipulation tends to vanish significantly when the notion of selfselectivity is imposed.
\end{abstract}

KEYWORDS: Voting rules, Self-selectivity, Stability, Manipulability, Probability.

JEL CLASSIFICATION: D72

\section{Introduction}

Voting rules aggregating individual preferences into a collective choice differ in their vulnerability to manipulation. Manipulating an election is for voters (individual voter or a coalition of voters) a way to announce non sincere individual preferences in order to achieve better voting result for themselves. We know from the well-known theorem of Gibbard-Satterthwaite [12, 29] that all voting rules, when choosing a single candidate for at least three candidates and without a dictator, are vulnerable to this type of strategic behavior. These studies have given rise to a number of extensions and generalizations of Gibbard-Satterthwaite theorem.

Many papers dealing with the probability of different voting rules to be manipulable under various definitions of manipulability was presented in the literature. This framework stipulates to introduce a certain measure of manipulability of a voting rule and a certain assumptions on the distribution of voter preferences. These manipulability measures were first introduced by Nitzan [28] for the case of individual manipulation and Lepelley and Mbih [23] for the case of coalitional manipulation. Other interesting manipulability measures were also presented by Aleskerov and Kurbanov [1], Kelly [17, 18] and Smith [31].

The question of the probability of manipulability was widely investigated by Favardin et al. [9], Lepelley and Mbih [24, 25], Favardin and Lepelley [8], Lepelley and Valognes [26] and Gehrlein and Lepelley [11]. The mean idea of these papers is to evaluate the proportion of preference profiles or voting situations that are not equilibria, that is the voting situations at which the voting rule taken into consideration is manipulable by an individual or a coalition of individuals. So, the vulnerability to individual or coalitional manipulability is used in order to

\footnotetext{
${ }^{*}$ Université de Lyon, Lyon, F-69007, France ; CNRS, GATE Lyon Saint-Etienne, Ecully, F-69130, France ; Université Jean Monnet, Saint-Etienne, F-42000, France. Tel.: + 33 (0)4 7742 19 60. Email: diss@gate.cnrs.fr.
} 
compare voting rules according to their sensitivity. However, no paper has tried to evaluate the probability of strategic manipulability of a self-selective voting rule.

Self-selectivity is a new principle introduced in the social choice literature. It is a desirable property of voting rules considered when individuals have to choose a voting rule in a set of voting rules. A voting rule is self-selective at some profile if, given this profile, there is no alternative rule that beats the given voting rule if the given voting rule is used to choose between the rules in the set. If a rule, which is not self-selective is used, then there will be a large enough group of voters who all prefer another voting rule to be successful in changing the rule. The notion of selfselectivity gives rise to another original concept when the given set of voting rules is considered. A set of voting rules is (weakly) stable if it always contains at least one self-selective rule at any profile or voting situation. In this case, if none of the voting rules in the set is self selective, the society could never be able to vote how to vote. These questions were first considered by Koray [19], Koray and Unel [20], Barberà and Jackson [3], Barberà and Bevià [2] and Houy [14, 15, 16]. Considering some probabilistic models, these questions were also investigated by Diss and Merlin [5] and Diss et al. [4].

The concepts of individual and coalitional manipulation can be extended quite naturally to the notion of self-selective voting rules. The vote will be on voting rules and, as in the basic framework of manipulation, it can be profitable for some voter at some voting situation to misrepresent his preferences on voting rules in order to have an outcome (voting rule) preferred to that resulting in the voting situation in which his vote reflects his true preferences. As a consequence, manipulating a self-selective voting rule means that this rule becomes not-selective after manipulation. This situation can lead to a serious paradox of instability if all the other considered voting rules are not self-selective after manipulation. This is equivalent to a situation in which it is impossible to directly or indirectly reach a self-selective voting rule if implementing any voting rule of the considered set. This problem can be solved by considering self-selectivity before and after manipulation. That is, when we consider the manipulation of a self-selective voting rule, the considered set of voting rules must contain at least one self-selective voting rule after manipulation. In other words, the set of voting rules must be stable after manipulation.

We reconsider in this paper the question of the probability of both individual and coalitional manipulability for three most commonly used voting rules in the literature: Borda rule, Copeland rule and Plurality rule. The main idea will be first to characterize the voting situations at which each voting rule is self-selective and vulnerable to manipulation by an individual or a coalition of individuals given that the set of the considered voting rules remains stable after manipulation. Secondly, the probabilities of the vulnerability are found using the Impartial and Anonymous Culture (IAC) assumption and the three voting rules Borda, Copeland and Plurality are compared taking into consideration these probabilities. The route followed in this paper concerns the impact of the self-selectivity and stability assumptions on the probability of vulnerability of each voting rule to both individual and coalitional manipulation.

The paper is organized as follows. The basic notions and assumptions used throughout this paper are introduced in section 2. For the Borda rule, the Copeland method and the Plurality rule, we analyze manipulation by a single voter in section 3 and section 4 is devoted to coalitional manipulation. Finally, section 5 presents our conclusions.

\section{Basic framework and definitions}

Consider $n$ voters, with cardinality $n \geq 2$, showing their true preferences on the set $X$ composed by Copeland method (Cop), Borda rule (Bor) and Plurality rule (Plu). Without ties, the six possible preference orderings over this set will be numbered in Table 1. This Table means that, for instance, $n_{1}$ individuals prefer Borda to Copeland, which is preferred to Plurality. A preference profile 
Table 1: The 6 preference rankings for 3 voting rules

$$
\begin{aligned}
& \text { Bor } \succ \text { Cop } \succ \text { Plu } \quad n_{1} \quad \text { Cop } \succ \text { Plu } \succ \text { Bor } \quad n_{4} \\
& \text { Bor } \succ P l u \succ C o p \quad n_{2} \quad P l u \succ B o r \succ C o p \quad n_{5} \\
& \text { Cop } \succ B o r \succ P l u \quad n_{3} \quad P l u \succ C o p \succ B o r \quad n_{6}
\end{aligned}
$$

is defined as a matrix consisting of $n$ vectors that represent voters' preferences by ordering the three alternatives (voting rules). A vector $\tilde{n}=\left(n_{1}, n_{2}, n_{3}, n_{4}, n_{5}, n_{6}\right)$ such that $\sum_{i} n_{i}=n$ defines a voting situation. The set of possible voting situations with size $n$ is then: $\mathcal{D}^{n}=\{\tilde{n}=$ $\left.\left(n_{1}, \ldots, n_{6}\right), \sum_{i=1}^{6} n_{i}=n, n_{i} \geq 0\right\}$. Consequently, we will define the voting rules on $\mathcal{D}=$ $\cup_{n=1}^{\infty} \mathcal{D}^{n}$ which will be a mapping from $\mathcal{D}$ into $2^{X} \backslash \emptyset$. In this paper, we assume that a voting rule is a social choice correspondence, that is, it associates to each voting situation at least one winning alternative (voting rule) in $X$. It means that each voting rule assigns to each voting situation a nonempty subset of $X$. Also, we shall assume that the collective decision for each voting situation only depends upon the restriction of the individual preferences.

We now introduce the voting rules used throughout this paper. If one candidate defeats all others head-to-head, that candidate is said to be the Condorcet winner. Of course, a candidate is a Condorcet loser if she loses all pairwise comparisons. For example, Bor is a Condorcet winner if and only if Bor wins against both Cop (i.e. $n_{1}+n_{2}-n_{3}-n_{4}+n_{5}-n_{6}>0$ ) and Plu (i.e. $n_{1}+n_{2}+n_{3}-n_{4}-n_{5}-n_{6}>0$ ). In rare occasions, each candidate is defeated by at least one other, so there is no Condorcet winner. One solution to such a paradox will be the Copeland method which generalizes the notion of pairwise majority rule, to suggest that the election winner should be the candidate that defeats the most other candidates by majority rule under pairwise comparison. For each pair of alternatives $a$ and $b$, let $N_{a, b}$ be the number of voter who prefer alternative $a$ to alternative $b$. In addition, let $T_{a, b}$ be the following function such that:

$$
T_{a, b}= \begin{cases}1, & \text { if } N_{a, b}>\frac{n}{2} \\ 0, & \text { if not }\end{cases}
$$

$T_{a, b}=1$ means that a majority of voters prefers alternative $a$ to alternative $b$. With this definition, one obtains the Copeland score of an alternative $a$ which is equal to the number of other alternatives that $a$ beats. This is equivalent to the following score:

$$
\text { Copeland Score }(a)=\sum_{b \in X} T_{a, b}
$$

where $X$ is the set of the available alternatives. Consequently, for each alternative $a$, the possible values of Copeland score ranges from 0 if $a$ is a Condorcet loser to $m-1$ if $a$ is a Condorcet winner. In other words, a Copeland winner is an alternative with maximal Copeland score and if there exists a Condorcet winner, this alternative is the unique Copeland winner. Notice that, in three candidate elections, the unique winner by Copeland rule must be the strict Condorcet winner, i.e. an alternative which has a strict majority against every other alternative. In addition, there must be a pairwise majority rule cycle if there is not a unique Copeland rule winner with an odd number of voters. That is, in some cases, it is necessary to use some tie-breaking procedure. The lexicographic order is the well-known example to break ties between two (or more) candidates. This assumption will be used throughout this paper.

Borda and Plurality rules belong to the well-known Weighted Scoring rules (or, simply, scoring rules) which can be defined as follows: each voter's ballot must be a vector that gives the number of points that the voter assigns to each candidate according to his or her position in the voter's 
preference. The vote vectors of all voters are summed and the winning candidate has the highest number of points. In a three-candidate election, each scoring rule can be defined by a vector $(2,1+\lambda, 0)$, where $-1 \leq \lambda \leq 1$. It means that each individual assigns $2,1+\lambda$ and 0 points respectively to each first, second and third placed candidate in this individual preference rankings. In this case, Borda rule and Plurality rule will be defined for $\lambda=0$ and $\lambda=-1$, respectively. Notice that, other classifications are possible in the sense that a linear transformation of a scoring rule does not change the outcome of the election.

The strategic manipulation of each self-selective voting rule in the set $X$ is the focus of our results in this paper. We use throughout this paper the definition given by Houy $[14,15,16]$.

Definition 1 Consider a set of voting rules $X$, and consider a given preference profile or voting situation on the available voting rules in $X$. A voting rule in $X$ is said to be self-selective if it chooses itself when it is used to decide between voting rules in $X$.

To illustrate this notion, let us consider an example of coalitional manipulation. Consider a society of 21 voters who have to choose a voting rule in the set $X=\{B o r, P l u, C o p\}$. The individual preferences are given in Table 2. For this example, Borda and Plurality rules are self-selective in the sense that the application of Borda gives out Borda as a result (the Borda scores of Borda, Copeland and Plurality respectively are 30,13 and 20) and the application of Plurality rule gives out itself (the Plurality scores of Borda, Copeland and Plurality respectively are 18, 4 and 20). In addition, Copeland is not self-selective since the application of Copeland method gives out Borda (the Copeland scores of Borda, Copeland and Plurality respectively are 2, 1 and 0). In this

Table 2: Self-selectivity and coalitional manipulation

\begin{tabular}{cc}
\hline Preference order & Number of voters \\
\hline Plu $\succ$ Bor $\succ$ Cop & 10 \\
Bor $\succ C o p \succ P l u$ & 9 \\
Cop $\succ$ Bor $\succ P l u$ & 2 \\
\hline
\end{tabular}

example, two voters (with preference $C o p \succ B o r \succ P l u$ ) are manipulating. In a sincere election, this type voters cast their votes for Copeland, but Plurality wins when we use Plurality. If this type voters anticipate this result, they can vote for Borda instead of Copeland; that is, they can vote with the preference Bor $\succ C o p \succ$ Plu. By voting with this preference, they guarantee that Borda, whom they prefer to Plurality, is elected.

Notice that the manipulation of each voting rule will lead to the fact that the considered voting rule becomes not self-selective after manipulation. For the modified example, Plurality rule is not self-selective since Plurality selects Borda rule (the Plurality scores of Borda, Copeland and Plurality respectively are 22, 0 and 20). Moreover, Borda remains self-selective since Borda rule selects itself (the Borda scores of Borda, Copeland and Plurality respectively are 32, 11 and 20). In addition, Copeland method remains not self-selective as Copeland is still selecting Borda (the Copeland scores of Borda, Copeland and Plurality remains respectively 2, 1 and 0 ). As said above, the notion of self-selectivity of voting rules is important for each society and still important after the manipulation. Indeed, this notion becomes fully operational only if there exists at least one selfselective rule at each profile. Since no voting rule remains self-selective after its manipulation, our objective will be to guarantee that each society must have a self-selective voting rule included in the set of voting rules $X$ with the new preferences of individuals if they misrepresent their preferences to manipulate. That is, in order to guarantee that the process of establishment of the rules remains in stable stage, the set of voting rules $X$ must be (weakly) stable. This is a meaningful condition on the set of valid voting rules which can be defined as follows (Houy $[14,15,16])$ : 
Definition 2 Consider a set of voting rules $X$, and consider a given preference profile on the available voting rules in $X$. The set $X$ is (weakly) stable if, at any profile, there exists at least one self-selective voting rule.

Of course, the set $X$ is unstable if, at any profile, there does not exist a self-selective voting rule in $X$. For preferences in Table 2, the manipulation of Plurality leads to the fact that this rule becomes not self-selective and gives out Borda rule which remains self-selective with the new preferences. That is, manipulation can be considered in this paper since it does not lead to temporary problems of instability. In this case, frequent manipulation cause that the general idea of a unique self-selective rule fails, nevertheless the social decision remains predictable in the sense that a self-selective rule will emerge.

The impact of both self-selectivity and stability on the probability of manipulation of each voting rule in the set $X$ will be our objective. In other words, the aim of this paper will be to reconsider the probability of strategic manipulation of each voting rule in the set $X$ when the considered voting rule is self-selective and when the set $X$ contains at least one self-selective voting rule after manipulation. This probability depends on the hypothesis that are set upon the behavior of the voters. Two models were widely studied in the literature: Impartial Culture (IC) and Impartial and Anonymous Culture (IAC). The main idea is the notion of equiprobability of profiles for IC and voting situations for IAC. In other words, Impartial Culture [10, 13] model assumes that each voter selects his preferences out of possible linear orders and each of preference profiles is equally likely. For the case of $m$ alternatives, the total number of different preference profiles is $(m !)^{n}$ and this model assumes that each of the $(m !)^{n}$ preference profiles is equally likely. In this paper, we will consider the IAC model which assumes that each voting situation has the same probability to occur. For $n$ agents and $m$ alternatives, the total number of voting situations is $C_{n+m !-1}^{n}$. For a specified $n$ in the case of 3 alternatives and using a simple calculation, the total number of voting situations is given by the fifth-degree polynomial $\psi(n)$ and therefore each voting situation $\tilde{n}=\left(n_{1}, n_{2}, n_{3}, n_{4}, n_{5}, n_{6}\right)$ will have the same probability to occur $\frac{1}{\psi(n)}$.

$$
\psi(n)=\frac{(n+5) !}{n ! 5 !}=\frac{1}{120} n^{5}+\frac{1}{8} n^{4}+\frac{17}{24} n^{3}+\frac{15}{8} n^{2}+\frac{137}{60} n+1
$$

\section{Self-selectivity and strategic individual manipulation}

Recall that, given a voting situation $\tilde{n}=\left(n_{1}, n_{2}, n_{3}, n_{4}, n_{5}, n_{6}\right)$, a voting rule is individually manipulable at $\tilde{n}$ if there exists a voter who, can benefit from a misrepresentation of her or his preference. We consider throughout this paper that the number of voters $n$ is supposed to be odd. This assumption is used in order to simplify the expression of some results of the paper. The propositions and numerical values for an even number of voters are obtained using the same techniques and are available upon request from the author. Notice that the conclusions obtained for an odd number of voters can be extended quite naturally to an even number of voters. Now, we can characterize all voting situations at which a self-selective Copeland rule is individually manipulable given that the set $X$ will contain at least one self-selective rule after manipulation. The same characterization is given for both Borda rule and Plurality rule. $\operatorname{Cop}(X, \tilde{n}), \operatorname{Bor}(X, \tilde{n})$ and $\operatorname{Plu}(X, \tilde{n})$ will denote respectively the Copeland winner, the Borda winner and the Plurality winner when the voting situation is $\tilde{n}$ and the set of available voting rules is $X$.

Lemma 1 Consider the voting situation $\tilde{n}=\left(n_{1}, n_{2}, n_{3}, n_{4}, n_{5}, n_{6}\right)$ and the set of available voting rules $X=\{B$ Bor, Cop, Plu $\}$. The Copeland rule is self-selective, individually manipulable at $\tilde{n}$ with guaranteed stability of $X$ if and only if the following conditions are satisfied: 
(i) $-n_{1}-n_{2}+n_{3}+n_{4}-n_{5}+n_{6}>0$ and $n_{1}+n_{2}+n_{3}-n_{4}-n_{5}-n_{6} \geq 1$ and $n_{1}-n_{2}+n_{3}+$ $n_{4}-n_{5}-n_{6}=1$ and $n_{1} \geq 1$ and $\left[-n_{1}-n_{2}+n_{5}+n_{6}>0\right.$ and $\left.-n_{3}-n_{4}+n_{5}+n_{6}>0\right]$; or

(ii) $-n_{1}-n_{2}+n_{3}+n_{4}-n_{5}+n_{6}>0$ and $n_{1}+n_{2}+n_{3}-n_{4}-n_{5}-n_{6} \geq 1$ and $n_{1}-$ $n_{2}+n_{3}+n_{4}-n_{5}-n_{6}=1$ and $n_{1} \geq 1$ and $\left[2 n_{1}+n_{2}+n_{3}-n_{4}-n_{5}-2 n_{6}>1\right.$ and $\left.n_{1}+2 n_{2}-n_{3}-2 n_{4}+n_{5}-n_{6}>-1\right]$.

Proof. Notice that the first part outside the bracketed list in (i) and (ii) can be considered as a particular case of Lemma 1 in Favardin et al. [9]. Consider first the situation corresponding to selfselectivity before manipulation. The Copeland rule is self-selective if and only if $\operatorname{Cop}(X, \tilde{n})=$ Cop. Thus, we must have $n_{3}+n_{4}+n_{6}>n_{1}+n_{2}+n_{5}$ and $n_{1}+n_{3}+n_{4}>n_{2}+n_{5}+n_{6}$. This is equivalent to the fact that $C o p$ is a Condorcet winner. In such a situation, no individual manipulation in favor of $\mathrm{Plu}$ can occur because a supporter of Plu cannot make Plu beat Cop in pairwise comparison. However, a supporter of Bor can make Bor the Copeland winner by modifying the majority relation between $C o p$ and Plu rules $\left(n_{1} \geq 1\right)$ in order to obtain a cycle and then breaking this cycle in favor of Bor instead of Cop. This strategy will have as a result the election of Bor if and only if Bor is preferred to Plu (this is equivalent to $n_{1}+n_{2}+n_{3}-n_{4}-n_{5}-$ $n_{6} \geq 1$ ) and Cop is preferred to Plu by one vote (this is equivalent to $n_{1}-n_{2}+n_{3}+n_{4}-n_{5}-n_{6}=$ 1). Nevertheless, given that the condition $n_{1}-n_{2}+n_{3}+n_{4}-n_{5}-n_{6}=1$ is included in the condition $n_{1}+n_{3}+n_{4}>n_{2}+n_{5}+n_{6}$, we will only keep the first one. In addition, we need that the set has at least one self-selective voting rule when the individual voter manipulates. This implies that Borda or Plurality need to be self-selective. It is equivalent to the part inside the bracketed list of each point of the Lemma. We know that an individual with preferences Bor $\succ C o p \succ P l u$ can manipulate the election by voting Bor $\succ P l u \succ C o p$. When this individual misrepresents her preferences, the new voting situation becomes $\tilde{n}=\left(n_{1}-1, n_{2}+1, n_{3}, n_{4}, n_{5}, n_{6}\right)$ and the Plurality rule is self-selective if and only if $-n_{1}-n_{2}+n_{5}+n_{6}>0$ and $-n_{3}-n_{4}+n_{5}+n_{6}>0$. This proves the necessity of (i). When Borda rule is self-selective, this implies $2 n_{1}+n_{2}+n_{3}-n_{4}-n_{5}-2 n_{6}>1$ and $n_{1}+2 n_{2}-n_{3}-2 n_{4}+n_{5}-n_{6}>-1$. This proves the necessity of (ii).

Now, we give the exact formulas of the probability of vulnerability of Copeland rule to individual manipulation when this rule is self-selective and the set of available voting rules $X$ remains stable after manipulation.

Proposition 1 Consider the voting situation $\tilde{n}=\left(n_{1}, n_{2}, n_{3}, n_{4}, n_{5}, n_{6}\right)$ with $n$ odd and the set of available voting rules $X=\{B o r, C o p, P l u\}$. Consider that Copeland rule is self-selective and that the set $X$ contains at least one self-selective voting rule after manipulation. The probability of vulnerability of Copeland rule to individual manipulation for $n \geq 13$ is given by:

- $\frac{5}{288} \frac{(n-1)\left(9 n^{3}+77 n^{2}+83 n-457\right)}{(n+1)(n+2)(n+3)(n+4)(n+5)}$ for $n=1 \bmod 12$

- $\frac{5}{288} \frac{(n-3)\left(9 n^{3}+95 n^{2}+291 n+333\right)}{(n+1)(n+2)(n+3)(n+4)(n+5)}$ for $n=3 \bmod 12$

- $\frac{5}{288} \frac{(n+7)\left(9 n^{3}+5 n^{2}-77 n-289\right)}{(n+1)(n+2)(n+3)(n+4)(n+5)}$ for $n=5 \bmod 12$

- $\frac{5}{288} \frac{(n+5)\left(9 n^{2}-22 n+1\right)}{(n+4)(n+3)(n+2)(n+1)} \quad$ for $n=7 \bmod 12$

- $\frac{5}{288} \frac{9 n^{3}+41 n^{2}-117 n-189}{(n+5)(n+4)(n+2)(n+1)} \quad$ for $n=9 \bmod 12$

- $\frac{5}{288} \frac{9 n^{3}+59 n^{2}-101 n-727}{(n+5)(n+4)(n+3)(n+2)} \quad$ for $n=11 \bmod 12$ 
Proof. In this paper, the proof of each proposition can be formulated as counting the exact number of integer solutions in the finite systems of linear constraints with rational coefficients in each corresponding Lemma. As recently pointed out by Lepelley et al. [22] and Wilson and Pritchard [33], the appropriate mathematical concepts to study such problems are polytopes and Ehrhart polynomials [6, 7]. These concepts were also used by the author in Diss et al. [4]. Using Parameterized Barvinok's algorithm (Verdoolaege et al. [32]), under the condition $\sum_{i=1}^{6} n_{i}=n$ and $n_{i} \geq 0$, the number of integer points inside system (i) in Lemma 1 is given by the Ehrhart polynomial $Y(n)$ for $n \geq 7$ as:

$$
\begin{aligned}
Y(n)= & \frac{5}{10368} n^{4}+\frac{1}{324} n^{3}+\left[0, \frac{-1}{576}, 0, \frac{-1}{576}, 0, \frac{-19}{1728}\right]_{n} n^{2}+\left[0, \frac{-7}{324}, 0, \frac{-1}{36}, 0, \frac{-23}{324}\right]_{n} n \\
& +\left[0, \frac{205}{10368}, 0, \frac{-3}{128}, 0, \frac{-595}{10368}\right]_{n}
\end{aligned}
$$

The bracketed list $\left[0, \frac{-1}{576}, 0, \frac{-1}{576}, 0, \frac{-19}{1728}\right]_{n}$ is a periodic number with period 6 which depends on the value of $n$. It is equivalent to $\frac{-1}{576}$ if $n=1 \bmod 6$ or $n=3 \bmod 6$, and $\frac{-19}{1728}$ if $n=5 \bmod 6$. It is also equal to 0 in the other cases. In addition, the number of integer points inside system (ii) in Lemma 1 is given by the Ehrhart polynomial $Z(n)$ for $n \geq 5$ as:

$$
Z(n)=\frac{1}{1152} n^{4}+\frac{1}{144} n^{3}+\frac{-1}{576} n^{2}+\frac{-1}{16} n+\left[0, \frac{65}{1152}, 0, \frac{-7}{128}, 0, \frac{-7}{128}\right]_{n}
$$

Recall that we need to find the number of integer points in the intersection of events (i) and (ii) and to subtract it from $Y(n)+Z(n)$. This number is given by the Ehrhart polynomial $I(n)$ for $n \geq 13$ as:

$$
\begin{aligned}
I(n)= & \frac{1}{20736} n^{4}+\frac{1}{5184} n^{3}+\left[0, \frac{-5}{1152}, 0, \frac{-5}{1152}, 0, \frac{-23}{3456}\right]_{n} n^{2}+\left[0, \frac{-31}{5184}, 0, \frac{-7}{576}, 0, \frac{-71}{5184}\right]_{n} n \\
& +\left[0, \frac{209}{20736}, 0, \frac{17}{256}, 0, \frac{3745}{20736}, 0, \frac{1505}{20736}, 0, \frac{1}{256}, 0, \frac{-143}{20736}\right]_{n}
\end{aligned}
$$

As a consequence, for $n \geq 13$, the cardinality of (i) and (ii) is given by $Y(n)+Z(n)-I(n)$ which will be divided by the total number of situations $\psi(n)$ in order to find the probability of vulnerability of Copeland rule to individual manipulation as a function of $n$. This proves the proposition 1. The corresponding probabilities are given in Table 3. Notice that for the case $n=5$ the probability of vulnerability of Copeland rule to individual manipulation is given by $\frac{Z(n)}{\psi(n)}$. In addition for $7 \leq n<13$, this probability is given by $\frac{Y(n)+Z(n)}{\psi(n)}$ in the sense that the intersection between (i) and (ii) is empty when $n<13$.

The same characterization can be found for Borda rule. Let $B(j)$ and $B^{\prime}(j)$ be the Borda score of alternative $j \in X$ before and after manipulation, respectively.

Lemma 2 Consider the voting situation $\tilde{n}=\left(n_{1}, n_{2}, n_{3}, n_{4}, n_{5}, n_{6}\right)$ and the set of available voting rules $X=\{B$ Bor, Cop, Plu $\}$. The Borda rule is self-selective, individually manipulable at $\tilde{n}$ with guaranteed stability of $X$ if and only if the following conditions are satisfied:

(i) $B(B o r)=B(C o p)$ and $B(B o r) \geq B(P l u)$ and $n_{6} \geq 1$ and $\left[\left(B^{\prime}(P l u)>B^{\prime}(B o r)\right.\right.$ and $\left.B^{\prime}(P l u)>B^{\prime}(C o p)\right)$ or $\left(B^{\prime}(C o p)>B^{\prime}(B o r)\right.$ and $\left.\left.B^{\prime}(C o p)>B^{\prime}(P l u)\right)\right]$; or

(ii) $B($ Bor $)=B(C o p)$ and $B(B o r) \geq B(P l u)$ and $B(C o p) \geq B(P l u)+1$ and $n_{3} \geq 1$ and $\left[\left(B^{\prime}(P l u)>B^{\prime}(B o r)\right.\right.$ and $\left.B^{\prime}(P l u)>B^{\prime}(C o p)\right)$ or $\left(B^{\prime}(C o p)>B^{\prime}(B o r)\right.$ and $\left.\left.B^{\prime}(C o p)>B^{\prime}(P l u)\right)\right]$; or

(iii) $B($ Bor $)=B(P l u)$ and $B(B o r) \geq B(C o p)$ and $n_{4} \geq 1$ and $\left[\left(B^{\prime}(P l u)>B^{\prime}(B o r)\right.\right.$ and $\left.B^{\prime}(P l u)>B^{\prime}(C o p)\right)$ or $\left(B^{\prime}(C o p)>B^{\prime}(B o r)\right.$ and $\left.\left.B^{\prime}(C o p)>B^{\prime}(P l u)\right)\right]$; or 
(iv) $B($ Bor $)=B(P l u)$ and $B(B o r) \geq B(C o p)$ and $B(P l u) \geq B(C o p)+1$ and $n_{5} \geq 1$ and $\left[\left(B^{\prime}(P l u)>B^{\prime}(B o r)\right.\right.$ and $\left.B^{\prime}(P l u)>B^{\prime}(C o p)\right)$ or $\left(B^{\prime}(C o p)>B^{\prime}(B o r)\right.$ and $\left.\left.B^{\prime}(C o p)>B^{\prime}(P l u)\right)\right]$.

Proof. Notice that the first part outside the bracketed list in (i), (ii), (iii) and (iv) can be considered as a particular case of Lemma 2 in Favardin et al. [9]. Under the first part of condition (i), we can easily check that a voter with preferences $P l u \succ C o p \succ B o r\left(n_{6} \geq 1\right)$ can give $C o p \succ P l u \succ$ $B o r$. This strategy increases $B(C o p)$ by one point and decreases $B(P l u)$ by one point. $B(B o r)$ remains the same since the position of Bor does not change. As a consequence, if $B(B o r)=$ $B(C o p)$ and $B(B o r) \geq B(P l u)$ and $n_{6} \geq 1$, the winner will be $C o p$ and Borda rule becomes manipulable. In addition, as said before, the manipulation of Borda will lead to the new voting situation $\left(n_{1}, n_{2}, n_{3}, n_{4}+1, n_{5}, n_{6}-1\right)$. In this case, Plurality is self-selective if and only if $B^{\prime}(P l u)>B^{\prime}(B o r)$ and $B^{\prime}(P l u)>B^{\prime}(C o p)$. In addition, Copeland is self-selective if and only if $B^{\prime}(C o p)>B^{\prime}(B o r)$ and $B^{\prime}(C o p)>B^{\prime}(P l u)$. This proves the necessity of (i). Now, the voter with the preference $C o p \succ B o r \succ P l u$ can also manipulate if Borda is self-selective. This voter can have the preference $C o p \succ P l u \succ B o r$. That is, B(Bor) decreases by one point, $B(P l u)$ increases by one point and $B(C o p)$ remains the same. Then, if $B(B o r)=B(C o p)$ and $B(B o r) \geq B(P l u)$ and $B(C o p) \geq B(P l u)+1$ and $n_{3} \geq 1$, manipulation leads to the fact that $C o p$ becomes the winner. This proves the necessity of (ii). Similarly, we can easily find conditions (iii) and (iv) by assuming that the Borda rule is manipulable in favor of Plurality rule.

The reader will easily check that, for example, under condition (i), $B(B o r)=B(C o p)$ and $B(B o r) \geq B(P l u)$ correspond to $2 n_{1}+2 n_{2}+n_{3}+n_{5}=2 n_{3}+2 n_{4}+n_{1}+n_{6}$ and $2 n_{1}+$ $2 n_{2}+n_{3}+n_{5} \geq 2 n_{5}+2 n_{6}+n_{4}+n_{2}$, respectively. In addition, after the manipulation, Plurality is self-selective if and only if $-n_{3}-n_{4}+n_{5}+n_{6}>2$ and $-n_{1}-n_{2}+n_{5}+n_{6}>1$. In addition, Copeland is self-selective if and only if $-n_{1}-n_{2}+n_{3}+n_{4}-n_{5}+n_{6}>0$ and $n_{1}-n_{2}+n_{3}+n_{4}-n_{5}-n_{6}>-2$. It defines the system of (in)equalities in the condition (i). We can easily find the remaining (in)equalities for each point of Lemma which are available upon request from the author. Now, we give the exact formulas of the probability of vulnerability of Borda rule to individual manipulation in our framework.

Proposition 2 Consider the voting situation $\tilde{n}=\left(n_{1}, n_{2}, n_{3}, n_{4}, n_{5}, n_{6}\right)$ with $n$ odd and the set of available voting rules $X=\{B o r, C o p, P l u\}$. Consider that Borda rule is self-selective and that the set $X$ contains at least one self-selective voting rule after manipulation. The probability of vulnerability of Borda rule to individual manipulation for $n \geq 10$ is given by:

- $\frac{5}{864} \frac{55 n^{4}+868 n^{3}+4266 n^{2}-19420 n+159383}{(n+1)(n+2)(n+3)(n+4)(n+5)}$ for $n=1 \bmod 12$

- $\frac{5}{864} \frac{55 n^{4}+868 n^{3}+4266 n^{2}-18396 n+175527}{(n+1)(n+2)(n+3)(n+4)(n+5)}$ for $n=3 \bmod 12$

- $\frac{5}{864} \frac{55 n^{4}+868 n^{3}+3930 n^{2}-16124 n+171271}{(n+1)(n+2)(n+3)(n+4)(n+5)}$ for $n=5 \bmod 12$

- $\frac{5}{864} \frac{55 n^{4}+868 n^{3}+4266 n^{2}-19420 n+160679}{(n+1)(n+2)(n+3)(n+4)(n+5)}$ for $n=7 \bmod 12$

- $\frac{5}{864} \frac{55 n^{4}+868 n^{3}+4266 n^{2}-18396 n+174231}{(n+1)(n+2)(n+3)(n+4)(n+5)}$ for $n=9 \bmod 12$

- $\frac{5}{864} \frac{55 n^{4}+868 n^{3}+3930 n^{2}-16124 n+167383}{(n+1)(n+2)(n+3)(n+4)(n+5)}$ for $n=11 \bmod 12$

The reader will easily check that the first case in both (iii) and (iv) are impossible. It is also possible to check that other cases are similar and will give the same Ehrhart polynomial. The proof is similar to the corresponding one of Copeland rule. However, there are 6 possible cases in this Lemma. That is, we must use the Sylvester's inclusion-exclusion principle (or, Sieve formula) 
in order to consider the different intersections between each case. $n \geq 10$ corresponds to the case in which each system and each intersection in the Lemma 2 is non empty. This allows us to have a general mathematical representation. Different representations are found for $n<10$. The exact formulas for each case in Lemma 2 and each intersection are available upon request from the author. The corresponding probabilities of the vulnerability of Borda rule to individual manipulation are given in Table 3. Now, let us give the same characterization for the Plurality rule.

Table 3: The probabilities of individual and collective manipulation of a self-selectivity voting rule as a function of the number of voters $n$.

\begin{tabular}{||c|ccc|ccc||}
\hline & \multicolumn{3}{|c|}{ Individual manipulation } & \multicolumn{3}{c||}{ Collective manipulation } \\
$n$ & Borda & Copeland & Plurality & Borda & Copeland & Plurality \\
\hline 3 & 0.0714 & 0 & 0 & 0 & 0 & 0 \\
5 & 0.0516 & 0.0040 & 0 & 0.0159 & 0.0119 & 0 \\
7 & 0.0379 & 0.0076 & 0.0215 & 0.0278 & 0.0202 & 0.0240 \\
9 & 0.0320 & 0.0075 & 0.0195 & 0.0340 & 0.0220 & 0.0280 \\
11 & 0.0268 & 0.0069 & 0.0133 & 0.0387 & 0.0263 & 0.0266 \\
13 & 0.0231 & 0.0068 & 0.0189 & 0.0430 & 0.0300 & 0.0354 \\
15 & 0.0204 & 0.0063 & 0.0178 & 0.0461 & 0.0312 & 0.0379 \\
17 & 0.0181 & 0.0058 & 0.0136 & 0.0487 & 0.0334 & 0.0374 \\
19 & 0.0164 & 0.0056 & 0.0159 & 0.0511 & 0.0355 & 0.0421 \\
21 & 0.0150 & 0.0052 & 0.0152 & 0.0530 & 0.0362 & 0.0437 \\
23 & 0.0137 & 0.0049 & 0.0124 & 0.0546 & 0.0376 & 0.0435 \\
25 & 0.0127 & 0.0046 & 0.0135 & 0.0561 & 0.0389 & 0.0464 \\
27 & 0.0118 & 0.0044 & 0.0131 & 0.0574 & 0.0394 & 0.0475 \\
29 & 0.0109 & 0.0041 & 0.0111 & 0.0585 & 0.0404 & 0.0474 \\
$\vdots$ & $\vdots$ & $\vdots$ & $\vdots$ & $\vdots$ & $\vdots$ & $\vdots$ \\
51 & 0.0063 & 0.0026 & 0.0082 & 0.0658 & 0.0454 & 0.0549 \\
$\vdots$ & $\vdots$ & $\vdots$ & $\vdots$ & $\vdots$ & $\vdots$ & $\vdots$ \\
101 & 0.0032 & 0.0014 & 0.0044 & 0.0713 & 0.0493 & 0.0598 \\
$\vdots$ & $\vdots$ & $\vdots$ & $\vdots$ & $\vdots$ & $\vdots$ & $\vdots$ \\
201 & 0.0016 & 0.0007 & 0.0024 & 0.0743 & 0.0514 & 0.0626 \\
$\vdots$ & $\vdots$ & $\vdots$ & $\vdots$ & $\vdots$ & $\vdots$ & $\vdots$ \\
501 & 0.0006 & 0.0003 & 0.0010 & 0.0762 & 0.0527 & 0.0644 \\
$\vdots$ & $\vdots$ & $\vdots$ & $\vdots$ & $\vdots$ & $\vdots$ & $\vdots$ \\
1001 & 0.0003 & 0.0002 & 0.0005 & 0.0769 & 0.0532 & 0.0650 \\
$\vdots$ & $\vdots$ & $\vdots$ & $\vdots$ & $\vdots$ & $\vdots$ & $\vdots$ \\
$\infty$ & 0 & 0 & 0 & 0.0775 & 0.0536 & 0.0656 \\
\hline
\end{tabular}

As in the case of Borda rule and Copeland rule, notice that the vulnerability of Plurality rule to manipulation (without self-selectivity consideration) was widely considered in the literature. The vulnerability of Plurality rule to individual manipulation can be found in Lepelley and Mbih [25]. Indeed, we adapt the proof to the case when individuals have to choose between voting rules in the set $X$ and when the rule under consideration is self-selective after manipulation. The situation after manipulation is easy to consider in order to guarantee that the set $X$ remains stable after manipulation. 
Lemma 3 Consider the voting situation $\tilde{n}=\left(n_{1}, n_{2}, n_{3}, n_{4}, n_{5}, n_{6}\right)$ and the set of available voting rules $X=\{B o r, C o p, P l u\}$. The Plurality rule is self-selective, individually manipulable at $\tilde{n}$ with guaranteed stability of $X$ if and only if the following conditions are satisfied:

(i) $n_{5}+n_{6}=n_{1}+n_{2}+1$ and $n_{5}+n_{6}>n_{3}+n_{4}$ and $n_{3} \geq 1$ and $\left[\left(n_{1}+2 n_{2}-n_{3}-2 n_{4}+n_{5}-n_{6}+\right.\right.$ $2>0$ and $\left.2 n_{1}+n_{2}+n_{3}-n_{4}-n_{5}-2 n_{6}+1>0\right)$ or $\left(-n_{1}-n_{2}+n_{3}+n_{4}-n_{5}+n_{6}-2>0\right.$ and $\left.\left.n_{1}-n_{2}+n_{3}+n_{4}-n_{5}-n_{6}>0\right)\right]$; or

(ii) $n_{5}+n_{6}=n_{3}+n_{4}+1$ and $n_{5}+n_{6}>n_{1}+n_{2}$ and $n_{1} \geq 1$ and $\left[\left(n_{1}+2 n_{2}-n_{3}-2 n_{4}+n_{5}-n_{6}-\right.\right.$ $2>0$ and $\left.2 n_{1}+n_{2}+n_{3}-n_{4}-n_{5}-2 n_{6}-1>0\right)$ or $\left(-n_{1}-n_{2}+n_{3}+n_{4}-n_{5}+n_{6}+2>0\right.$ and $\left.\left.n_{1}-n_{2}+n_{3}+n_{4}-n_{5}-n_{6}>0\right)\right]$.

Proof. In order to have $P l u(X, \tilde{n})=P l u$, we must have $n_{5}+n_{6}>n_{1}+n_{2}$ and $n_{5}+n_{6}>n_{3}+n_{4}$. In such a situation, the only individual manipulation in favor of $B$ or will be from an individual with the preferences Cop $\succ B o r \succ P l u$ who can vote Bor $\succ C o p \succ P l u$. This individual $\left(n_{3} \geq 1\right)$ can increase the Plurality score of Bor by one, and decrease the Plurality score of $C o p$ by one, and make Bor the Plurality winner if $n_{5}+n_{6}=n_{1}+n_{2}+1$. As a consequence, the condition $n_{5}+n_{6}>n_{1}+n_{2}$ is not required. In addition, after manipulation the new voting situation will be $\tilde{n}=\left(n_{1}+1, n_{2}, n_{3}-1, n_{4}, n_{5}, n_{6}\right)$. In such a situation, Borda rule is self-selective if and only if $n_{1}+2 n_{2}-n_{3}-2 n_{4}+n_{5}-n_{6}+2>0$ and $2 n_{1}+n_{2}+n_{3}-n_{4}-n_{5}-2 n_{6}+1>0$. In addition, Copeland rule is self-selective if and only if $-n_{1}-n_{2}+n_{3}+n_{4}-n_{5}+n_{6}-2>0$ and $n_{1}-n_{2}+n_{3}+n_{4}-n_{5}-n_{6}>0$. This proves the necessity of (i). The same characterization can be found in (ii) when the manipulation is in favor of Cop.

Proposition 3 Consider the voting situation $\tilde{n}=\left(n_{1}, n_{2}, n_{3}, n_{4}, n_{5}, n_{6}\right)$ with $n$ odd and the set of available voting rules $X=\{B o r, C o p, P l u\}$. Consider that Plurality rule is self-selective and that the set $X$ contains at least one self-selective voting rule after manipulation. The probability of vulnerability of Plurality rule to individual manipulation for $n \geq 19$ is given by:

- $\frac{5}{1296} \frac{133 n^{4}+392 n^{3}+3606 n^{2}-21448 n+48421}{(n+1)(n+2)(n+3)(n+4)(n+5)}$ for $n=1 \bmod 12$

- $\frac{5}{1296} \frac{(n-3)\left(133 n^{3}+1011 n^{2}+2511 n+1377\right)}{(n+1)(n+2)(n+3)(n+4)(n+5)}$ for $n=3 \bmod 12$

- $\frac{5}{1296} \frac{(n-5)\left(133 n^{3}+837 n^{2}+2031 n+679\right)}{(n+1)(n+2)(n+3)(n+4)(n+5)} \quad$ for $n=5 \bmod 12$

- $\frac{5}{1296} \frac{133 n^{4}+392 n^{3}+3606 n^{2}-21448 n+48421}{(n+1)(n+2)(n+3)(n+4)(n+5)}$ for $n=7 \bmod 12$

- $\frac{5}{1296} \frac{133 n^{3}+213 n^{2}-1161 n-2673}{(n+5)(n+4)(n+2)(n+1)} \quad$ for $n=9 \bmod 12$

- $\frac{5}{1296} \frac{133 n^{3}+39 n^{2}-2193 n-7283}{(n+5)(n+4)(n+3)(n+2)} \quad$ for $n=11 \bmod 12$

The proof of this proposition is similar to the propositions above. Sieve formula was also considered in this case. The exact formulas for each case in Lemma 3 and each intersection are available upon request from the author. Notice that different representations are found for $n<19$ depending on the domain of each case and each intersection in Lemma 3. Table 3 and Figure 1 (for $n=3, \ldots, 51$ ) display the values of the probability of individual manipulation as a function of the number of voters $n$. As in the case without self-selectivity, the vulnerability to individual manipulation for each voting rule in the set $X$ decreases as the number $n$ of voters increases. This probability will tend to 0 when the number of voters is sufficiently large. In addition, for some values of $n$, the Borda rule appears to be more vulnerable than the Plurality rule, which is more vulnerable than the Copeland method. However, for $n \geq 25$, the Plurality rule is more vulnerable than 
the Borda rule which remains more vulnerable than the Copeland method. For example, in the case of $n=1001$, the probability of vulnerability to individual manipulation is equal to 0.0003 , 0.0002 and 0.0005 for Borda rule, Copeland rule and Plurality rule, respectively. Furthermore, the probability of individual manipulation tends to vanish significantly when the notions of selfselectivity and stability are imposed. The numerical results displayed in Table 3 in this paper, Table 1 in Favardin et al. [9] and Table 1 in Gehrlein and Lepelley [11] allow us to distinguish results for cases with and without self-selectivity consideration. For instance, for the case of $n=51$, these tables show that the probability of vulnerability of individual manipulation is equal to 0.0382 for the Borda rule, 0.0214 for the Copeland rule and 0.0490 for the Plurality rule in the standard case without self-selectivity. These probabilities are respectively equivalent to 0.0063 , 0.0026 and 0.0082 in our new framework of self-selectivity consideration. In other words, the probabilities in the standard case are greater than the new framework by a factor of 6.1 for the Borda rule, 8.2 for the Copeland method and 6.0 for the Plurality rule. Finally, unlike to the case without self-selectivity consideration, we can notice that the probability of vulnerability to individual manipulation in our new framework is null for $n=3$ for the Copeland method and for $n=3$ and $n=5$ for the Plurality rule.

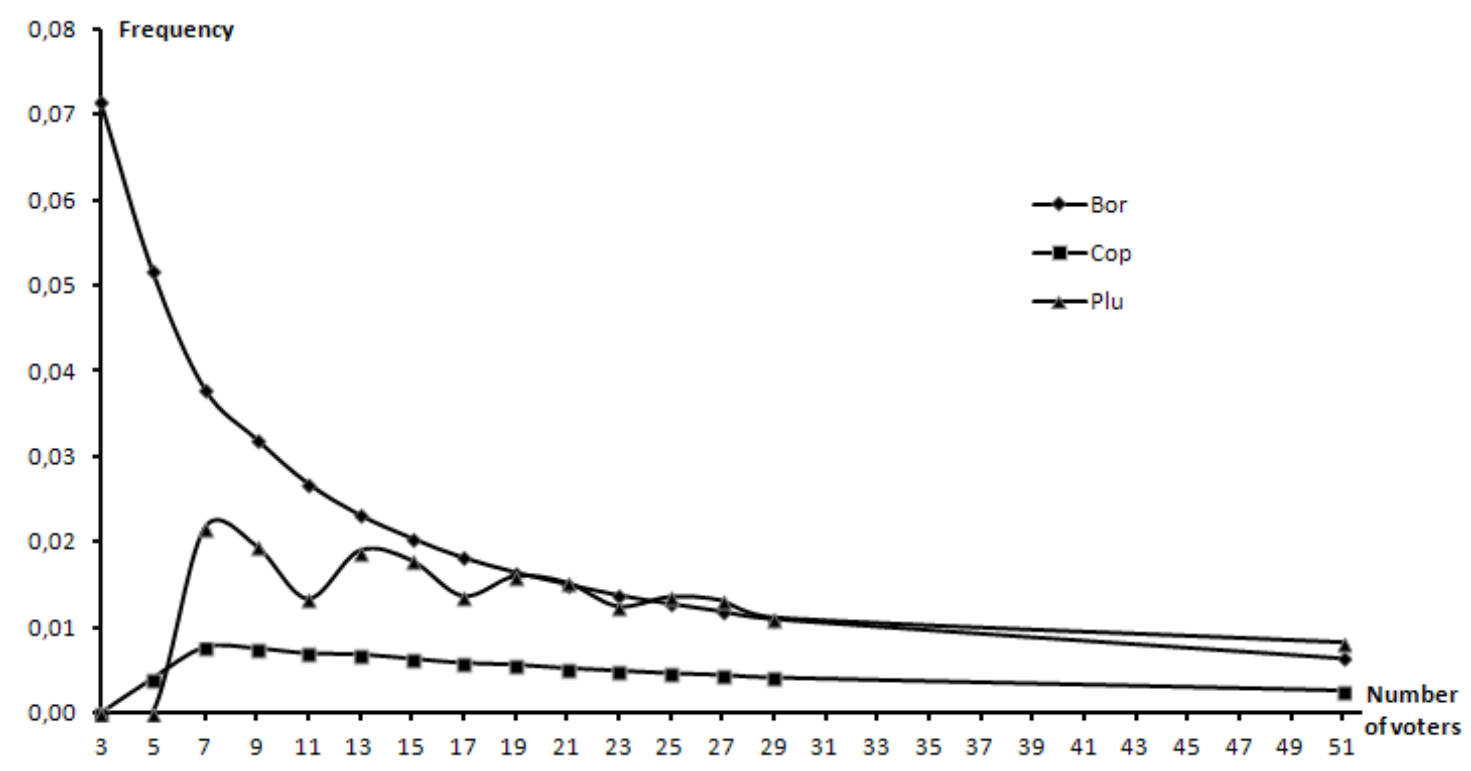

Figure 1: Frequencies of individual manipulation of a self-selective voting rule for $n=3, \ldots, 51$.

\section{Self-selectivity and strategic coalitional manipulation}

Recall that, a coalition of voters is any non empty subset of the set containing all voters. Given a voting situation $\tilde{n}=\left(n_{1}, n_{2}, n_{3}, n_{4}, n_{5}, n_{6}\right)$, a voting rule is said to be coalitionally manipulable at $\tilde{n}$ if there exists a coalition of agents of the same type who can all benefit from a misrepresentation of their preferences. We can characterize all voting situations at which a self-selective Copeland rule (Borda rule, Plurality rule) is coalitionally manipulable given that the set $X=\{B o r, C o p, P l u\}$ will contain at least one self-selective rule after manipulation. As said before, the proof of each characterization will be based on the results in the literature concerning the manipulation of each voting rule in three-candidate election. We adapt these results for the case when individuals have to choose between voting rules under some conditions. 
Lemma 4 Consider the voting situation $\tilde{n}=\left(n_{1}, n_{2}, n_{3}, n_{4}, n_{5}, n_{6}\right)$ and the set of available voting rules $X=\{B o r, C o p, P l u\}$. The Copeland rule is self-selective, coalitionally manipulable at $\tilde{n}$ with guaranteed stability of $X$ if and only if the following conditions are satisfied:

(i) $-n_{1}-n_{2}+n_{3}+n_{4}-n_{5}+n_{6}>0$ and $n_{1}-n_{2}+n_{3}+n_{4}-n_{5}-n_{6}>0$ and $n_{1}+n_{2}-n_{3}-n_{4}+$ $n_{5}+n_{6} \geq 1$ and $n_{1}+n_{2}+n_{3}-n_{4}+n_{5}-n_{6} \geq 1$ and $\left[n_{1}+n_{2}+n_{3}-n_{4}+n_{5}-2 n_{6}>0\right.$ and $\left.2 n_{1}+2 n_{2}-n_{3}-2 n_{4}+2 n_{5}-n_{6}>0\right]$; or

(ii) $-n_{1}-n_{2}+n_{3}+n_{4}-n_{5}+n_{6}>0$ and $n_{1}-n_{2}+n_{3}+n_{4}-n_{5}-n_{6}>0$ and $n_{1}+n_{2}-$ $n_{3}-n_{4}+n_{5}+n_{6} \geq 1$ and $n_{1}+n_{2}+n_{3}-n_{4}+n_{5}-n_{6} \geq 1$ and $\left[-n_{3}-n_{4}+n_{6}>0\right.$ and $\left.-n_{1}-n_{2}-n_{5}+n_{6}>\right] 0$.

Proof. Notice that the first part outside the bracketed list in (i) and (ii) can be considered as a particular case of Lemma 1 in Favardin et al. [9]. For the set $X=\{B o r, C o p, P l u\}$, in order to have $\operatorname{Cop}(X, \tilde{n})=C o p$, the rule $\operatorname{Cop}$ must be the Condorcet winner. This is equivalent to the first two inequalities in (i). In such situation, as in the case of Lemma 1, no manipulation can occur in favor of Plu. By contrast, the voters preferring Bor can modify the majority relation between $C o p$ and $P l u$ rules in order to obtain a cycle to make Bor the Copeland winner. Now, suppose that voters with preferences Bor $\succ C o p \succ P l u$ decide to vote for $P l u$ in the pairwise comparison between $C o p$ and Plu. In addition, suppose that voters with preferences Plu $\succ$ Bor $\succ C o p$ decide to vote for Bor in the pairwise comparison between Bor and Plu. As a result, the number of individuals voting for Plu against $C o p$ will be equal to $n_{1}+n_{2}+n_{5}+n_{6}$ and the number of individuals voting for $B$ or against $P l u$ will be equal to $n_{1}+n_{2}+n_{3}+n_{5}$. Then, if $n_{1}+n_{2}+n_{5}+n_{6}>n_{3}+n_{4}$ (i.e. $n_{1}+n_{2}-n_{3}-n_{4}+n_{5}+n_{6} \geq 1$ ) and $n_{1}+n_{2}+n_{3}+n_{5}>n_{4}+n_{6}$ (i.e. $n_{1}+n_{2}+n_{3}-n_{4}+n_{5}-n_{6} \geq 1$ ), then a cycle will occur and Bor will be the winner. In addition, we need that the set has at least one self-selective voting rule when the individual voter manipulates. This implies that Borda or Plurality need to be self-selective. With the new voting situation $\tilde{n}=\left(0, n_{1}+n_{2}+n_{5}, n_{3}, n_{4}, 0, n_{6}\right)$, Borda is self-selective if and only if $n_{1}+n_{2}+n_{3}-n_{4}+n_{5}-2 n_{6}>0$ and $2 n_{1}+2 n_{2}-n_{3}-2 n_{4}+2 n_{5}-n_{6}>0$. This proves the necessity of (i). When Plurality rule is self-selective, this implies $-n_{3}-n_{4}+n_{6}>0$ and $-n_{1}-n_{2}-n_{5}+n_{6}>0$. This proves the necessity of (ii).

Proposition 4 Consider the voting situation $\tilde{n}=\left(n_{1}, n_{2}, n_{3}, n_{4}, n_{5}, n_{6}\right)$ with $n$ odd and the set of available voting rules $X=\{B$ or $, C o p, P l u\}$. Consider that Copeland rule is self-selective and that the set $X$ contains at least one self-selective voting rule after manipulation. The probability of vulnerability of Copeland rule to coalitional manipulation for $n \geq 7$ is given by:

- $\frac{1}{2592} \frac{(n-1)\left(139 n^{3}+329 n^{2}+629 n+919\right)}{(n+4)(n+3)(n+2)(n+1)} \quad$ for $n=1 \bmod 6$

- $\frac{1}{2592} \frac{(n-3)\left(139 n^{3}+885 n^{2}+1701 n+1755\right)}{(n+5)(n+4)(n+1)(n+2)}$ for $n=3 \bmod 6$

- $\frac{1}{2592} \frac{139 n^{4}+746 n^{3}+24 n^{2}-4154 n-4435}{(n+5)(n+2)(n+3)(n+4)}$ for $n=5 \bmod 6$

Using Parameterized Barvinok's algorithm, the number of integer points inside systems (i) and (ii) in Lemma 4 is given by an Ehrhart polynomial. Notice that the number of integer points in the intersection of (i) and (ii) is equal to 0 . Notice also that conditions in (i) of Lemma 4 give solutions if and only if $n \geq 5$ while conditions in (ii) admit solutions if and only if $n \geq 7$. The proof is similar to the Propositions 1, 2 and 3 and is available upon request from the author.

In the following Lemma, which analyses the coalitional manipulation of the Borda rule, let $B(i, j)=B(i)-B(j)$ be the difference between the Borda score of alternative $i \in X$ and $j \in X$ before manipulation. $B(j)$ and $B^{\prime}(j)$ still stand for the Borda score of alternative $j \in X$ before and after manipulation, respectively. 
Lemma 5 Consider the voting situation $\tilde{n}=\left(n_{1}, n_{2}, n_{3}, n_{4}, n_{5}, n_{6}\right)$ and the set of available voting rules $X=\{B o r, C o p, P l u\}$. The Borda rule is self-selective, coalitionally manipulable at $\tilde{n}$ with guaranteed stability of $X$ if and only if the following conditions are satisfied:

(i) $B(B o r)>B(C o p)$ and $B(B o r)>B(P l u)$ and $B(C o p, P l u)+2 n_{6} \geq n_{3}$ and $B(B o r, C o p)<$ $n_{3}+n_{6}$ and $B^{\prime}(C o p)>B^{\prime}(B o r)$ and $B^{\prime}(C o p)>B^{\prime}(P l u)$; or

(ii) $B(B o r)>B(C o p)$ and $B(B o r)>B(P l u)$ and $n_{3}>B(C o p, P l u)+2 n_{6} \geq 0$ and $B(B o r, C o p)<B(C o p, P l u)+3 n_{6}$ and $B^{\prime}(C o p)>B^{\prime}(B o r)$ and $B^{\prime}(C o p)>B^{\prime}(P l u)$; or

(iii) $B(B o r)>B(C o p)$ and $B(B o r)>B(P l u)$ and $B(P l u, C o p)+2 n_{4} \geq n_{5}$ and $B(B o r, P l u)<$ $n_{4}+n_{5}$ and $B^{\prime}(P l u)>B^{\prime}(B o r)$ and $B^{\prime}(P l u)>B^{\prime}(C o p)$; or

(iv) $B(B o r)>B(C o p)$ and $B(B o r)>B(P l u)$ and $n_{5} \geq B(P l u, C o p)+2 n_{4} \geq 0$ and $B(B o r, P l u)<B(P l u, C o p)+3 n_{4}-1$ and $B^{\prime}(P l u)>B^{\prime}(B o r)$ and $B^{\prime}(P l u)>$ $B^{\prime}(C o p)$.

Proof. Conditions (i) and (ii) (respectively (iii) and (iv)) deal with the case where the Borda rule is manipulable by a coalition of voters in favor of Copeland (respectively Plurality). Notice that the first part (before manipulation) in (i), (ii), (iii) and (iv) can be considered as a particular case of Lemma 4 in Favardin et al. [9]. We adapt this result to the case when individuals have to choose between voting rules under some conditions. In addition, it is easy to check that if the 3-type voters with preferences $C o p \succ B o r \succ P l u$ and the 6-type voters with preferences Plu $\succ C o p \succ B o r$ manipulate according to the case (i) or (ii), Plurality rule can not be selfselective after manipulation. Otherwise, if the 4-type voters and the 5-type voters manipulate according to the case (iii) or (iv), Copeland rule can not be self-selective after manipulation. The exact proof of this Lemma are available under request from the author.

The reader will easily check that, for example, under condition (i), $B(C o p, P l u)+2 n_{6} \geq n_{3}$ and $B(B o r, C o p)<n_{3}+n_{6}$ correspond to $n_{1}-n_{2}+n_{3}+n_{4}-2 n_{5}+n_{6} \geq 0$ and $n_{1}+$ $2 n_{2}-2 n_{3}-2 n_{4}+n_{5}-2 n_{6}<0$, respectively. In addition, after the manipulation, both voters with preferences $\mathrm{Cop} \succ \mathrm{Bor} \succ \mathrm{Plu}$ and Plu $\succ C o p \succ B o r$ can misrepresent their sincere preferences and vote $C o p \succ P l u \succ B o r$. In this case, the new voting situation will be $\tilde{n}=\left(n_{1}\right.$, $\left.n_{2}, 0, n_{3}+n_{4}+n_{6}, n_{5}, 0\right)$. In this case, Copeland rule is self-selective if and only if $B^{\prime}(C o p)>$ $B^{\prime}(B o r)$ and $B^{\prime}(C o p)>B^{\prime}(P l u)$ which is equivalent to $-n_{1}-n_{2}+n_{3}+n_{4}-n_{5}+n_{6}>0$ and $n_{1}-n_{2}+n_{3}+n_{4}-n_{5}+n_{6}>0$. We can easily find the remaining inequalities which are available upon request from the author. Finally, we give the characterization of vulnerability of Plurality rule to coalitional manipulation in our new framework.

Lemma 6 Consider the voting situation $\tilde{n}=\left(n_{1}, n_{2}, n_{3}, n_{4}, n_{5}, n_{6}\right)$ and the set of available voting rules $X=\{B o r, C o p, P l u\}$. The Plurality rule is self-selective, coalitionally manipulable at $\tilde{n}$ with guaranteed stability of $X$ if and only if the following conditions are satisfied:

(i) $n_{5}+n_{6}>n_{1}+n_{2}$ and $n_{5}+n_{6}>n_{3}+n_{4}$ and $n_{1}+n_{2}+n_{3}>n_{5}+n_{6}$ and $\left[\left(n_{1}+2 n_{2}+n_{3}-2 n_{4}+\right.\right.$ $n_{5}-n_{6}>0$ and $\left.2 n_{1}+n_{2}+2 n_{3}-n_{4}-n_{5}-2 n_{6}>0\right)$ or $\left(-n_{1}-n_{2}-n_{3}+n_{4}-n_{5}+n_{6}>0\right.$ and $\left.\left.n_{1}-n_{2}+n_{3}+n_{4}-n_{5}-n_{6}>0\right)\right]$; or

(ii) $n_{5}+n_{6}>n_{1}+n_{2}$ and $n_{5}+n_{6}>n_{3}+n_{4}$ and $n_{1}+n_{3}+n_{4}>n_{5}+n_{6}$ and $\left[\left(-n_{1}+2 n_{2}-n_{3}-\right.\right.$ $2 n_{4}+n_{5}-n_{6}>0$ and $\left.n_{1}+n_{2}+n_{3}-n_{4}-n_{5}-2 n_{6}>0\right)$ or $\left(n_{1}-n_{2}+n_{3}+n_{4}-n_{5}+n_{6}>0\right.$ and $\left.\left.n_{1}-n_{2}+n_{3}+n_{4}-n_{5}-n_{6}>0\right)\right]$.

Proof. In order to have $P l u(X, \tilde{n})=P l u$, we must have $n_{5}+n_{6}>n_{1}+n_{2}$ and $n_{5}+n_{6}>n_{3}+n_{4}$. In such a situation, the only coalitional manipulation in favor of $B$ or will be from individuals with 
the preferences Cop $\succ B o r \succ P l u$ who can vote Bor $\succ C o p \succ P l u$. Those individuals can increase the Plurality score of $B$ or by $n_{3}$, and decrease the Plurality score of $C o p$ by $n_{3}$, and make $B$ or the Plurality winner if $n_{1}+n_{2}+n_{3}>n_{5}+n_{6}$. In addition, after manipulation the new voting situation will be $\tilde{n}=\left(n_{1}+n_{3}, n_{2}, 0, n_{4}, n_{5}, n_{6}\right)$. In such a situation, Borda rule is self-selective if and only if $n_{1}+2 n_{2}+n_{3}-2 n_{4}+n_{5}-n_{6}>0$ and $2 n_{1}+n_{2}+2 n_{3}-n_{4}-n_{5}-2 n_{6}>0$. In addition, Copeland rule is self-selective if and only if $-n_{1}-n_{2}-n_{3}+n_{4}-n_{5}+n_{6}>0$ and $n_{1}-n_{2}+n_{3}+n_{4}-n_{5}-n_{6}>0$. This proves the necessity of (i). The same characterization can be found in (ii) when the manipulation is in favor of Cop.

Using the same approach as in the other propositions, the number of integer points inside systems in Lemma 5 and 6 will be given by the Ehrhart polynomial. However, the complexity of the conditions characterizing those situations at which the Borda rule and the Plurality rule are manipulable by a coalition of voters makes impossible the derivation of a general mathematical representation for the vulnerability of each voting rule to coalitional manipulation under some conditions in our framework. This is due in large part to the fact that the periodicity of each Ehrhart polynomial is very large in some cases. Fortunately, when the number of voters is given, exact numerical values can be found for each case. Notice that, for large electorates ( $n$ tends to $\infty$ ), the corresponding probabilities are found by taking into account the coefficient of the higher degree in each Ehrhart polynomial and the coefficient $\left(\frac{1}{120}\right)$ of the higher degree in $\psi(n)$.

Table 3 and Figure 2 (for $n=3, \ldots, 51$ ) display the values of the probability of coalitional manipulation as a function of the number of voters $n$. As in the case without self-selectivity, it turns out that the vulnerability to coalitional manipulation for each voting rule in the set $X$ increases as the number $n$ of voters increases. When the number of voters is sufficiently large, this probability will be equal to $0.0775,0.0536$ and 0.0656 for the Borda rule, the Copeland method and the Plurality rule, respectively. In addition, unlike to the case of individual manipulation, whatever the value of $n$, the Borda rule appears to be significantly more vulnerable than the Plurality rule, which is more vulnerable than the Copeland method. For example, in the case of $n=51$, the probability of vulnerability to coalitional manipulation is equal to $0.0658,0.0454$ and 0.0549 for Borda rule, Copeland rule and Plurality rule, respectively. As in the case of individual manipulation, the probability of coalitional manipulation tends to vanish significantly when the notions of self-selectivity and stability are imposed. The numerical results displayed in Table 3 in this paper, Table 1 in Favardin et al. [9] and Table 2 in Gehrlein and Lepelley [11] allow us to compare the two cases. For instance, for large electorates, these tables show that the probability of vulnerability of coalitional manipulation is equal to 0.5025 for the Borda rule, 0.2500 for the Copeland rule and 0.2917 for the Plurality rule in the standard case without self-selectivity. These probabilities are respectively equivalent to $0.0775,0.0536$ and 0.0656 in our new framework of self-selectivity. That is, the probabilities in the standard case are greater than the new framework by a factor of 6.5 for the Borda rule, 4.7 for the Copeland method and 4.4 for the Plurality rule. Finally, unlike to the case without self-selectivity consideration, we can notice that the probability of vulnerability to individual manipulation in our new framework is null for $n=3$ for the Copeland method and the Borda rule and for $n=3$ and $n=5$ for the Plurality rule.

\section{Conclusion and further research}

The main aim of this paper was to reconsider the impact of the self-selectivity and stability assumptions on the probability of vulnerability of each voting rule to strategic manipulation. It seams that the probability of individual and coalitional manipulation tends to vanish significantly when these notions are imposed to voting rules. In addition, the Borda rule appears to be more vulnerable to individual and coalitional manipulation than the Copeland rule and the Plurality rule.

Notice that, as we have analyzed a particular case of Impartial and Anonymous Culture (IAC), 


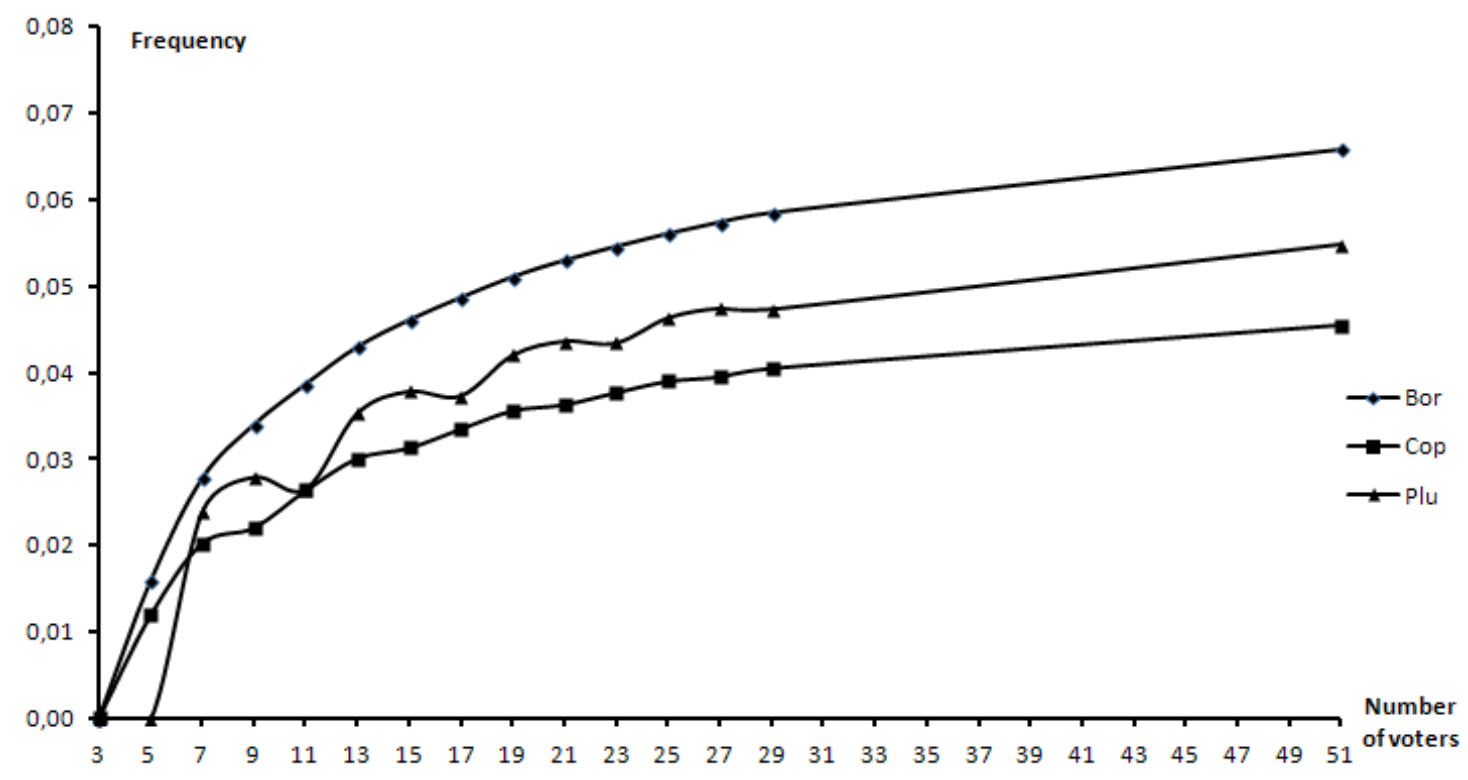

Figure 2: Frequencies of coalitional manipulation of a self-selective voting rule for $n=3, \ldots, 51$.

the extension of these results to Impartial Culture or other probabilistic hypothesis [21] remains open. In addition, the extension of this paper to other voting rules remains possible. In such situation, considering Iterative scoring rules could be interesting to study. This voting system generalize the scoring voting rules to more than one round. That is, for the three-alternative case, after the first voting round, the two alternatives with the highest scores go to the run-off. The candidate who is able to defeat the other one on the basis of their pairwise comparison is then declared as the winner. Also, one can think of studying the case of general scoring rule. Given that, in a three-candidate election, each scoring rule can be defined by a vector $(2,1+\lambda, 0)$, looking for the value of $\lambda$ which reduces the probability of the self-selective scoring rule $\lambda$ to be vulnerable to manipulation could be another way to follow.

Notice that the literature in self-selectivity and stability gives rise to another possibility which could be explored: Consequentialism property $[4,19,20]$. In such a situation, voters vote on a given set composed by alternatives which is similar to the basic framework of voting theory. In addition, we assume that voters have intrinsic preferences over the voting rules which depend on the results of the vote on alternatives. In other words, individuals rank the available voting rules in accordance with what they choose from the set of alternatives. A possible route could concern the impact of the consequentialism assumption on the probability of vulnerability of each voting rule to individual and coalitional manipulation.

Other types of manipulation can be considered. In fact, in some situations, candidates can have interest to exit the choice set [30]. Following the same way of studying the manipulation of voters by using probability models, we could look at the manipulability of each voting rule when the manipulation is based on candidates who prefer to exit the choice set. It should be noted that the way in which we measure manipulability by counting manipulable profiles or voting situations is not the only possible one. In fact, we could also analyse the minimal number [27] of profiles or voting situations at which each voting rule is manipulable. Many other possibilities can also be considered. 


\section{Acknowledgements}

I am indebted to Richard Baron for helpful comments on the earlier version of this paper.

\section{References}

[1] F. Aleskerov and E. Kurbanov. Degree of manipulability of social choice procedures. In Alkan et al. (eds.) Current Trends in Economics. Springer, Berlin Heidelberg New York, 1999.

[2] S. Barberà and S. Beviá. Self-selection consistent functions. Journal of Economic Theory, 105:263-277, 2002.

[3] S. Barberà and M.O. Jackson. Choosing how to choose: Self-stable majority rules and constitutions. The Quarterly Journal of Economics, 119(3):1011-1048, 2004.

[4] M. Diss, A. Louichi, V. Merlin, and H. Smaoui. An example of probability computations under the IAC assumption: The stability of scoring rules. Mathematical Social Sciences, 64(1):57-66, 2012.

[5] M. Diss and V. Merlin. On the stability of a triplet of scoring rules. Theory and Decision, 69:289-316, 2010.

[6] E. Ehrhart. Sur les polyèdres rationnels homothétiques à n dimensions. C. R. Acad. Sci. Paris, 254:616-618, 1962.

[7] E. Ehrhart. Sur un problème de géométrie diophantienne linéaire. PhD thesis, J R A Math, 226:1-49, 1967.

[8] P. Favardin and D. Lepelley. Some further results on the manipulability of social choice rules. Social Choice and Welfare, 26:485-509, 2006.

[9] P. Favardin, D. Lepelley, and J. Serais. Borda rule, Copeland method and strategic manipulation. Rev. Econ. Design, 7:213-228, 2002.

[10] W.V. Gehrlein and P.C. Fishburn. Condorcet's paradox and anonymous preference profiles. Public Choice, 26:1-18, 1976.

[11] W.V. Gehrlein and D. Lepelley. On some limitations of the median voting rule. Public Choice, 117:177-190, 2003.

[12] A.F. Gibbard. Manipulation of voting schemes: a general result. Econometrica, 41:587-601, 1973.

[13] G.T. Guilbaud. Les théories de l'intérêt général et le problème logique de l'agrégation. Economie Appliquée, 5:501-584, 1952.

[14] N. Houy. Dynamics of stable sets of constitutions. Mimeo, 2003.

[15] N. Houy. A note on the impossibility of a set of constitutions stable at different levels. Mimeo, 2004.

[16] N. Houy. De la stabilité des procédures et constitutions en théorie du choix social. $P h D$ Thesis, Paris 1, 2005. 
[17] J. Kelly. Minimal manipulability and local strategy-proofness. Social Choice and Welfare, 5:81-85, 1988.

[18] J. Kelly. Almost all social choice rules are highly manipulable, but few aren't. Social Choice and Welfare, 10:161-175, 1993.

[19] S. Koray. Self-selective social choice functions verify Arrow and Gibbard-Satterthwaite theorems. Econometrica, 68(4):981-995, 2000.

[20] S. Koray and B. Unel. Charactrization of self-selective social choice functions on the topsonly domain. Social Choice and Welfare, 20:495-507, 2003.

[21] J.-F. Laslier. In Silico voting experiments. In J.-F. Laslier and R. Sanver (Eds.), Handbook of approval voting. Heidelberg: Springer-Verlag, 2010.

[22] D. Lepelley, A. Louichi, and H. Smaoui. On Ehrhart polynomials and probability calculations in voting theory. Social Choice and Welfare, 30:363-383, 2008.

[23] D. Lepelley and B. Mbih. The proportion of coalitionally unstable situations under the plurality rule. Economics Letters, 24:311-315, 1987.

[24] D. Lepelley and B. Mbih. The vulnerability of four social choice rules to coalitional manipulation of preferences. Social Choice and Welfare, 11:253-265, 1994.

[25] D. Lepelley and B. Mbih. Strategic manipulation in committees using the plurality rule: Alternative concepts and frequency calculations. Group Decision and Negotiation, 6:119$138,1996$.

[26] D. Lepelley and F. Valognes. Voting rules, manipulability and social homogeneity. Public Choice, 116:165-184, 2003.

[27] S. Maus, H. Peters, and T. Storcken. Minimal manipulability: anonymity and unanimity. Social Choice and Welfare, 29:247-269, 2007.

[28] S. Nitzan. The vulnerability of point-voting schemes to preference variation and strategic manipulation. Public Choice, 47:349-370, 1985.

[29] M.A. Satterthwaite. Strategyproofness and Arrow's conditions: existence and correspondences for voting procedures and social welfare functions. Journal of Economic Theory, 10:187-217, 1975.

[30] J. Serais. Sensivity to strategic candidacy by exiting for scoring rules and Copeland. University of Caen Basse-Nomrnadie, unpubliched paper.

[31] D. Smith. Manipulability measures of common social choice functions. Social Choice and Welfare, 16:639-661, 1999.

[32] S. Verdoolaege, R. Seghir, K. Beyls, V. Loechner, and M. Bruynooghe. Analytical computation of Ehrhart polynomials: Enabling more compiler analysis and optimizations. In Proceedings of International Conference on Compilers, Architecture, and Synthesis for Embedded Systems. Washington D.C., pages 248-258, 2004.

[33] M.C. Wilson and G. Pritchard. Probability calculations under the IAC hypothesis. Mathematical Social Sciences, 54:244-256, 2007. 